\title{
Contamination: a comparison of 2 personal protective systems
}

\author{
Jorge E. Zamora, John Murdoch, Brian Simchison, Andrew G. Day
}

$\infty \quad$ See related article page 263

\section{ABSTRACT}

Background: The purpose of this study was to examine the difference in self-contamination rates and levels of contact and droplet protection associated with enhanced respiratory and contact precautions (E-RCP) and a personal protective system that included a full body suit, personal protective equipment and a powered air-purifying respirator (PAPR).

Methods: In this prospective, randomized, controlled crossover study, 50 participants donned and removed E-RCP and PAPR in random order. Surrogate contamination consisted of fluorescein solution and ultraviolet (UV) light-detectable paste, which was applied after each ensemble was donned. A blinded evaluator inspected participants for contamination using a UV lamp after removal of each ensemble. Areas of contamination were counted and measured in square centimetres. Donning and removal violations were recorded. The primary end point was the presence of any contamination on the skin or base clothing layer.

Results: Participants wearing E-RCP were more likely to experience skin and base-clothing contamination; their contamination episodes measuring $\geq 1 \mathrm{~cm}^{2}$ were more frequent, and they had larger total areas of contamination (all $p<$ 0.0001 ). The anterior neck, forearms, wrists and hands were the likeliest zones for contamination. Participants donning PAPR committed more donning procedure violations ( $p=$ $0.0034)$. Donning and removing the PAPR system took longer than donning and removing E-RCP garments ( $p$ $<0.0001)$.

Interpretation: Participants wearing E-RCP were more likely to experience contamination of their skin and base clothing layer. Those wearing PAPR required significantly more time to don and remove the ensemble and violated donning procedures more frequently.

CMAJ 2006;175(3):249-54

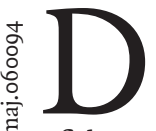
iseases transmitted via aerosols or respiratory droplets, the threat of emerging infectious diseases and the prospect of bioterrorism have become part of the new reality for health care workers. ${ }^{1}$ The optimum protective system for preventing disease transmission during aerosol-generating medical procedures (such as endotracheal intubation and fibreoptic bronchoscopy) involving patients with febrile respiratory illnesses has yet to be determined. Recent directives from the Ontario Ministry of Health and LongTerm Care (MOHLTC) stipulate the use of, at a minimum, enhanced respiratory and contact precautions (E-RCP), or the use of a personal protective system that combines a powered air-purifying respirator (PAPR) with other protective clothing. ${ }^{2}$ The US Center for Disease Control and Prevention (CDC) has recommended the use of personal protective equipment (PPE) when performing or assisting with aerosolgenerating procedures involving patients with SARS. ${ }^{3}$

Different personal protective systems have specific donning and removal procedures and might also offer different levels of protection from aerosolized droplets. The PAPR system requires many more steps for donning and removal than E-RCP or PPE. Protective systems that are more complicated to don and remove may expose personnel to an increased risk of self-contamination. The failure of a personal protective system may be associated with health consequences for frontline health care workers. SARS transmission has occurred despite the use of droplet, contact and airborne precautions. ${ }^{4,5} \mathrm{~A}$ potential explanation for some episodes of "through-precautions" transmission is the possibility of contamination during removal of protective clothing. ${ }^{6,7}$ At present, one study ${ }^{8}$ has been published that specifically examined through-gown contamination; another ${ }^{9}$ examined the different levels of protection associated with specific facial protective equipment systems. No data have been reported that address the question of self-contamination during the removal of personal protective clothing used in a health care setting.

Our study was performed to examine the difference in selfcontamination rates and the level of contact and droplet protection associated with E-RCP and the PAPR system.

\section{Methods}

After obtaining approval from the Queen's University Health Sciences and Affiliated Teaching Hospitals Reasearch Ethics Board, we recruited 50 participants for the study from among the resident and attending staff of the Department of Anesthesiology and practising and student members of the Department of Respiratory Therapy at Kingston General Hospital. They were informed of the purpose of the study, and each gave written consent.

Equipment for the E-RCP and PAPR systems is listed in Table I. The CDC recommendations have omitted a head cov- 
Table 1: Equipment list for the 2 protective-clothing systems compared

\begin{tabular}{|c|c|c|c|}
\hline \multicolumn{2}{|c|}{ System } & \multirow[b]{2}{*}{ Item } & \multirow[b]{2}{*}{ Manufacturer (location) } \\
\hline $\mathrm{E}-\mathrm{RCP}$ & PAPR & & \\
\hline & $\sqrt{ }$ & Tyvek hood & 3M (St. Paul, Minn.) \\
\hline$\sqrt{ }$ & $\sqrt{ }$ & Bouffant hair cover & $\begin{array}{l}\text { Prime Line Medical Products } \\
\text { (Edmonton, Alta.) }\end{array}$ \\
\hline \multirow[t]{2}{*}{$\sqrt{ }$} & $\sqrt{ }$ & Economy impact goggle & Spartan (Taiwan) \\
\hline & $\sqrt{ }$ & Air-mate breathing tube & 3M (Berkshire, United Kingdom) \\
\hline \multirow[t]{2}{*}{$\sqrt{ }$} & & Face shield & Splash Shield (Uniontown, Penn.) \\
\hline & $\sqrt{ }$ & HEPA filter unit & 3M (St. Paul, Minn.) \\
\hline \multirow[t]{7}{*}{$\sqrt{ }$} & $\sqrt{ }$ & \multicolumn{2}{|c|}{ N95 mask - any of several models: } \\
\hline & & 8210 & 3M (St. Paul) \\
\hline & & $1860 \mathrm{~s}$ & 3M (St. Paul) \\
\hline & & PFR95 & Kimberly-Clark (Roswell, Geo.) \\
\hline & & 7210 & Northern Safety (Frankfort, NY) \\
\hline & & 695 & Alpha Protech (North Salt Lake, Utah) \\
\hline & & Gloves & \\
\hline \multirow[t]{2}{*}{$\sqrt{ }$} & $\sqrt{ }$ & Nonlatex & SensiCare (Caledonia, Mich.) \\
\hline & $\sqrt{ }$ & Latex surgical & MicroTouch (Dothan, Ala.) \\
\hline \multirow[t]{3}{*}{$\sqrt{ }$} & $\sqrt{ }$ & Latex & AMD (Lachine, Que.) \\
\hline & $\sqrt{ }$ & Tyvek coveralls with hood & Lakeland Industries (Decatur, Ala.) \\
\hline & $\sqrt{ }$ & Tyvek boot covers & Lakeland Industries (Decatur) \\
\hline$\sqrt{ }$ & $\sqrt{ }$ & $\begin{array}{l}\text { Astound impervious } \\
\text { surgical gown }\end{array}$ & Cardinal Health (McGraw Park, IIl.) \\
\hline
\end{tabular}

Note: $\mathrm{E}-\mathrm{RCP}=$ enhanced respiratory and contact precautions, PAPR = powered air-purifying respirator, HEPA = high-efficiency particulate air.

ering as part of their PPE and state that goggles alone can be worn for eye protection. Because the MOHLTC directives stipulate that a head covering, goggles and a face shield must be worn, they were included in E-RCP for this study (Fig. I, Table I). The instructions used for donning and removal of E-RCP were an enhanced version of those set out in the MOHLTC directives. The PAPR system in use at our centre has outer and inner protective layers (Fig. 2).

Participants wore operating-room scrub attire as their base clothing layer. For study purposes, a towel was worn around the neck to protect it during the contamination procedure; this towel does not form part of the protective clothing systems recommended by MOHLTC or CDC. It was placed before donning protective clothing and was worn by members of both study groups. In the crossover design, participants were initially assigned by coin toss to don either the PAPR system or E-RCP garb, then the other personal protective system immediately after completing the removal protocol for the first. All participants were assessed with an ultraviolet (UV) lamp (Burton Medical Products, Van Nuys, Calif.) before donning the protective clothing in order to ensure that no UV fluorescence was present. All traces of surrogate contamination were removed before commencing the process for the second personal protective system. Participants were instructed to don and remove their protective systems at a pace that would allow them to follow correct donning and removal pro- cedures. Participants donning and removing ERCP followed written instructions only. While donning and removing the PAPR system, however, they were coached by a respiratory therapist referring tp written instructions. Both sets of instructions are available online (at www .cmaj.ca/cgi/content/full/175/3/249/DCI) as Appendix I and Appendix 2, respectively.

After donning protective clothing, participants were "contaminated" with a fluorescein solution ( $\mathrm{I} \mathrm{mL}$ of a $25 \%$ solution in $100 \mathrm{~mL}$ of sterile water). A Devilbiss atomizer (model DVI5-RD, Sunrise Medical Products, Carlsbad, Calif.) was used to apply $5 \mathrm{~mL}$ of solution to each participant's front face shield and torso. "Invisible" Detection Paste (I5 mL; Sirchie, Youngsville, NC) was applied from the forearms to the elbow and to the palmar aspects of participants' hands. The paste becomes visible when viewed under UV light.

All participants were timed and videotaped while donning and removing the protective clothing. For participants wearing the PAPR system, timing was paused after removal of the outer protective layer to allow for contamination assessment. Timing ceased when the participants removed the final item of protective clothing.

A donning or removal violation was defined as having occurred if a participant performed a manoeuvre out of sequence, touched an item of clothing out of sequence, tore an item of protective clothing, or touched any body part other than an item of protective clothing before performing the final handwashing step of the protocol.

After removal of the outer protective layer of the PAPR system, participants were assessed by an unblinded observer using a UV lamp. Then, an evaluator blinded to the participants' protective system inspected all areas of subjects' base clothing layer (including the neck-protection towel) and any exposed skin. Contamination of a neck-protection towel was recorded as contamination of the anterior or posterior neck. Areas of contamination were measured.

The primary end point of this study was the presence of contamination on any base clothing or exposed skin. Secondary end points included contamination of the inner layer of the PAPR system, area size on clothing or skin that underwent contamination, number of donning and removal violations, and time required to don and remove the protective systems. Demographic end points consisted of age, height, weight, sex, health care specialty and prior training in the donning and removal of the PAPR system.

Contamination outcomes were compared between systems with the Mainland-Gart test. ${ }^{10}$ It was applied by determining, for each subject, which period had a greater area of contamination, and then applying Fisher's exact test to determine if the period with worse contamination was related to the treatment sequence. Thus, subjects with no contamina- 
tion in either period were excluded. This method is appropriate for crossover studies where a period (learning) effect is possible and the number of subjects in each sequence is unequal. ${ }^{11,12}$ The Mainland-Gart test result is undefined if either period had no event; therefore, in cases where only 1 period had any contamination, we replaced it with McNemar's test. Protocol violations were compared in a similar manner. Donning and removal times were compared between systems with the standard method for 2-period crossover studies with continuous outcomes and a possible learning effect. ${ }^{10}$ The area of contamination among subjects with contamination was compared between the 2 systems with the exact Wilcoxon-MannWhitney test. For this tertiary outcome, appropriate methods for paired data were not applicable since the subjects with contamination were not the same for both systems. All $p$-value calculations were 2-sided; no adjustment was made for multiplicity of end points.

Our study used a convenience sample of 50 clinicians tested once on each system. If we assume that $80 \%$ of the subjects in each sequence are contaminated during the use of 1 system at least, and among those contaminated the probability of having more contamination with E-RCP is $75 \%$, then the Mainland-Gart test would provide $87 \%$ power at a 2 sided $\alpha$ of 0.05 .

\section{Results}

Demographic characteristics of the study participants are shown in Table 2.

\section{Contamination}

When the base clothing layer and exposed skin were examined, participants wearing E-RCP were more likely to experience contamination of any size, contamination with an area of $1 \mathrm{~cm}^{2}$ or more, and a larger total area of contamination when it was present (all $p<0.0001$; Table 3. Individual data are presented in Appendix 3, available online at www.cmaj.ca/cgi /content/full/175/3/249/DCI). The anterior neck and the forearms, hands, and wrists were the zones most likely to be contaminated (Fig. 3). When the anterior and posterior neck areas were excluded from the analysis, participants wearing E-RCP were still more likely to experience contamination of any size, contamination with an area of $1 \mathrm{~cm}^{2}$ or more (both $p$ $<$ o.oooI) and a larger total area of contamination $(p=0.013)$.

When contamination of the PAPR inner layer (Fig. 2, right panel) was compared to that of the E-RCP base layer or exposed skin (Table 4), participants wearing E-RCP were more likely to experience contamination of any size, contamination with an

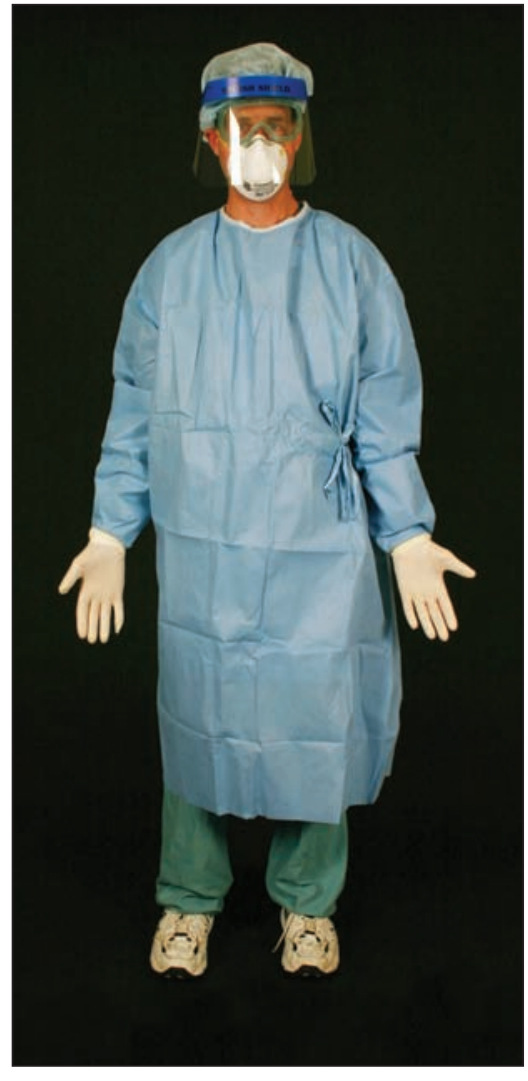

Fig. 1: Enhanced respiratory and contact precautions (E-RCP), familiar to most health care workers. The towel used for neck protection was omitted for illustrative purposes.

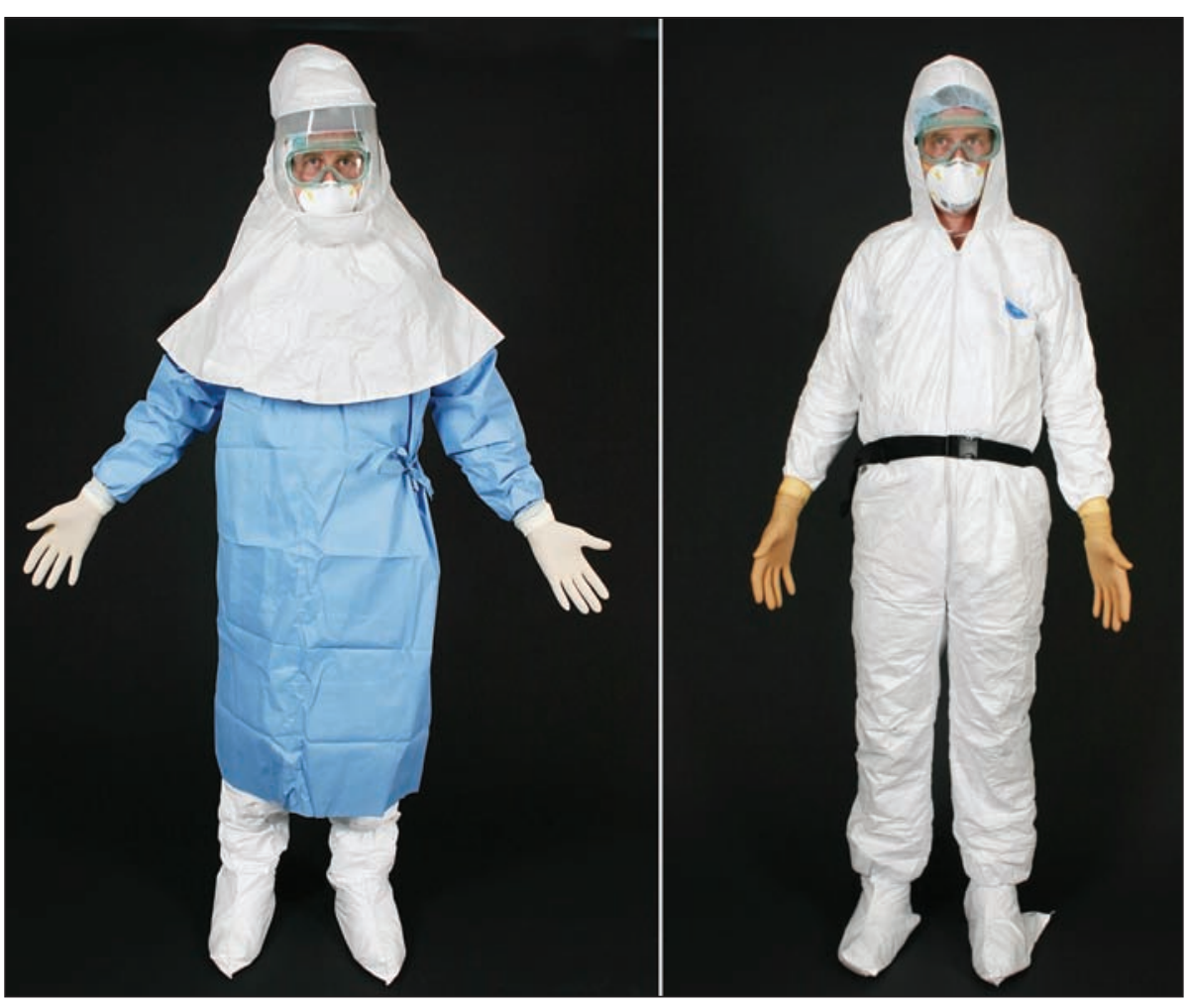

Fig. 2: The other system studied, named for its powered air-purifying respirator (PAPR), has 2 protective layers, shown above. The outer layer (left panel) consists of a hood, fluidresistant surgical gown, shoe covers and 2 pairs of fitted surgical gloves. The inner (right) includes a hooded coverall and shoe covers, PAPR power unit, N95 mask, goggles, bouffant hair cover, and 1 pair of fitted surgical gloves. Contamination assessment for this layer was performed with the PAPR power unit and fitted surgical gloves removed. The towel worn to protect the neck has been omitted for illustrative purposes. 
area of $1 \mathrm{~cm}^{2}$ or more, and a larger total area of contamination (all $p<0.000 \mathrm{I}$ ). Participants in E-RCP were again more likely to experience contamination of any size and contamination with an area of $1 \mathrm{~cm}^{2}$ or more (both $p<0.000 \mathrm{I}$ ) at the anterior neck. When the anterior and posterior neck were excluded from analysis, differences in contamination between the E-RCP base layer and the PAPR inner layer were nonsignificant.

\section{Donning and removal protocol violations}

Procedure violations were made by 15 participants during the donning of the PAPR system, versus 2 by those donning ERCP garb ( $p=0.003$; Table 5$)$. Group differences during protective clothing removal were not significant. In terms of multiple violations, 2 subjects removing E-RCP committed 2 violations; among those wearing PAPR, 4 committed 2 don-

Table 2: Characteristics of the study populations

\begin{tabular}{|c|c|c|}
\hline \multirow[b]{2}{*}{ Characteristic } & \multicolumn{2}{|c|}{ Order of system worn, mean (SD)* } \\
\hline & $\begin{aligned} \mathrm{PAPR} & =>\mathrm{E}-\mathrm{RCP} \\
n & =27\end{aligned}$ & $\begin{aligned} \mathrm{E}-\mathrm{RCP} & =>\mathrm{PAPR} \\
n & =23\end{aligned}$ \\
\hline Age, yr & $34.3 \quad(8.7)$ & $36.8 \quad(9.8)$ \\
\hline Height, $\mathrm{cm}$ & $171.8 \quad(8.1)$ & 172.3 \\
\hline Weight, kg & $76.3(16.7)$ & $71.3(13.0)$ \\
\hline Male-to-female ratio & $16: 11$ & $11: 12$ \\
\hline Anesthetists:RTs & $19: 08$ & $10: 13$ \\
\hline Any prior PAPR training, $n(\%)$ & $15(56)$ & $18(78)$ \\
\hline
\end{tabular}

Note: $\mathrm{SD}=$ standard deviation, $\mathrm{E}-\mathrm{RCP}=$ enhanced respiratory and contact precautions, PAPR = powered air-purifying respirator, RTs = respiratory therapists.

*Except as otherwise indicated. ning violations and 1 committed 2 removal violations. All other participants who violated donning or removal protocols did so only once.

\section{Timing}

On average, participants donning PAPR required 6 minutes, I7 seconds (range 222-517 s), whereas those donning E-RCP required I minute, 58 seconds (range $64-178 \mathrm{~s} ; p<0.000 \mathrm{I}$ ). Participants removing PAPR required 7 minutes, 32 seconds (range 296-667 s), whereas those removing E-RCP required 2 minutes, I5 seconds (range $78-2$ II s; $p<0.000$ I).

\section{Interpretation}

This study shows that a significantly higher number of participants wearing E-RCP experienced contamination, compared with those wearing the PAPR system. All but 2 participants using E-RCP experienced some degree of contamination at their anterior neck. This appeared to be primarily due to the fluorescein aerosol solution directed at participants' face shields and upper body, as opposed to the detection paste applied to their forearms and hands. When both the anterior and posterior neck were excluded from statistical analysis, subjects wearing E-RCP were still more likely to experience contamination, particularly of their forearms, wrists and hands.

Several reports have already been made of HCWs who wore PPE similar to that recommended by the CDC in highrisk situations yet still contracted SARS. ${ }^{4,5}$ Our study proposes a potential mechanism by which through-precautions transmission of diseases spread by aerosols or respiratory droplets might occur. Most recommendations for decontamination after wearing protective clothing stipulate handwashing. It is conceivable that, despite handwashing, HCWs could proceed

Table 3: Contamination data for skin and the base layer of clothing worn under the PAPR and E-RCP personal protective systems

\begin{tabular}{|c|c|c|c|c|c|c|c|c|c|}
\hline \multirow[b]{2}{*}{ Location } & \multicolumn{3}{|c|}{ Contamination, PAPR system } & \multicolumn{3}{|c|}{ Contamination, E-RCP } & \multicolumn{2}{|c|}{$p$, Mainland-Gart test* } & \multirow{2}{*}{$\begin{array}{c}p \\
\text { value, } \\
\text { WMW } \\
\text { test‡ }\end{array}$} \\
\hline & $\begin{array}{l}\text { Any } \\
n(\%)\end{array}$ & $\begin{array}{c}\geq 1 \mathrm{~cm}^{2} \\
n(\%)\end{array}$ & $\begin{array}{c}\text { Area, } \mathrm{cm}^{2} \\
\text { mean } n(\mathrm{SD})\end{array}$ & $\begin{array}{l}\text { Any } \\
n(\%)\end{array}$ & $\begin{array}{c}\geq 1 \mathrm{~cm}^{2} \\
n(\%)\end{array}$ & $\begin{array}{c}\text { Area, } \mathrm{cm}^{2} \\
\text { mean } n(\mathrm{SD})\end{array}$ & $\begin{array}{l}\text { Actual } \\
\text { areas }\end{array}$ & $\begin{array}{c}\text { Areas } \\
\geq 1 \mathrm{~cm}^{2} \dagger\end{array}$ & \\
\hline Face & 0 & 0 & NA & $2(4)$ & $1(2)$ & $1.1(0.6)$ & 1 & $1^{*}$ & Und. \\
\hline Back of head & 0 & 0 & NA & 0 & 0 & NA & Und. & Und. & Und. \\
\hline Anterior neck & $3(6)$ & $2(4)$ & $1.5(0.9)$ & $48(96)$ & $48(96)$ & $76.5(54.4)$ & $<0.001$ & $<0.001$ & $<0.001$ \\
\hline Forearms, hands or wrists & $9(18)$ & $7(14)$ & $1.8(1.8)$ & $38(76)$ & $35(70)$ & $6.5(7.6)$ & $<0.001$ & $<0.001$ & 0.015 \\
\hline Anterior torso, anterior upper arms & 0 & 0 & NA & $5(10)$ & $4(8)$ & $6.3(6.2)$ & $0.06^{*}$ & $0.13^{*}$ & Und. \\
\hline Back and posterior upper arms & 0 & 0 & NA & $1(2)$ & $1(2)$ & 7.0 & $1^{*}$ & $1^{*}$ & Und. \\
\hline Anywhere below beltline & 0 & 0 & NA & $1(2)$ & 0 & 0.5 & $1^{*}$ & Und. & Und. \\
\hline Total & $13(26)$ & $10(20)$ & $1.7(1.5)$ & $48(96)$ & $48(96)$ & $82.8(54.0)$ & $<0.001$ & $<0.001$ & $<0.001$ \\
\hline
\end{tabular}

Note: $\mathrm{E}-\mathrm{RCP}=$ enhanced respiratory and contact precautions, PAPR $=$ powered air-purifying respirator, WMW $=$ Wilcoxon-Mann-Whitney test, $\mathrm{NA}=$ not applicable, Und. = undefined.

${ }^{*}$ Except where McNemar's test was used, as indicated with asterisks. The Mainland-Gart test was used when contamination occurred during both periods. McNemar's test was substituted when only 1 period had any contamination. Neither test was applied when there was no contamination in either period.

tRepeat analysis, in which areas smaller than $1 \mathrm{~cm}^{2}$ in area were counted as $0 \mathrm{~cm}$.

$\ddagger$ The Wilcoxon-Mann-Whitney test was used to compare the area of contamination for subjects with some contamination. Since these contaminated subjects were different for each system, a test for paired data was not used. 
to touch other areas of their skin or clothing that have not been adequately decontaminated and then could infect themselves by touching their exposed mucus membranes.

Although the PAPR system offered superior protection, it required more time to don and remove it. Despite coaching by another person, a significantly higher number of participants committed violations while donning the PAPR system. Although prior training in the use of personal protective clothing systems might be expected to help address this important issue, our data provided no suggestion of an association between previous PAPR training, time required to don or remove protective clothing systems, donning or removal pro- cedure violations, or the amount of contamination of participants' base clothing or skin.

This study does have several limitations. Fluorescein solution and detection paste, and the areas to which they were applied, were chosen a priori to simulate worst-case viral contamination from respiratory droplets and direct patient contact, respectively. In addition, the definition of "significant contamination area" is unknown; we therefore analyzed our data by treating any contamination as significant. It is notable that, when we repeated our analysis, treating only contamination areas of greater than $1 \mathrm{~cm}^{2}$ as clinically significant, the difference between the 2 systems persisted.


Fig. 3: Examples of contamination of the forearm, wrist and hand (left panel) and neck of a study participant wearing enhanced respiratory and contact precautions (E-RCP).

Table 4: Comparison of contamination of PAPR inner-layer with E-RCP base-layer clothing and exposed skin

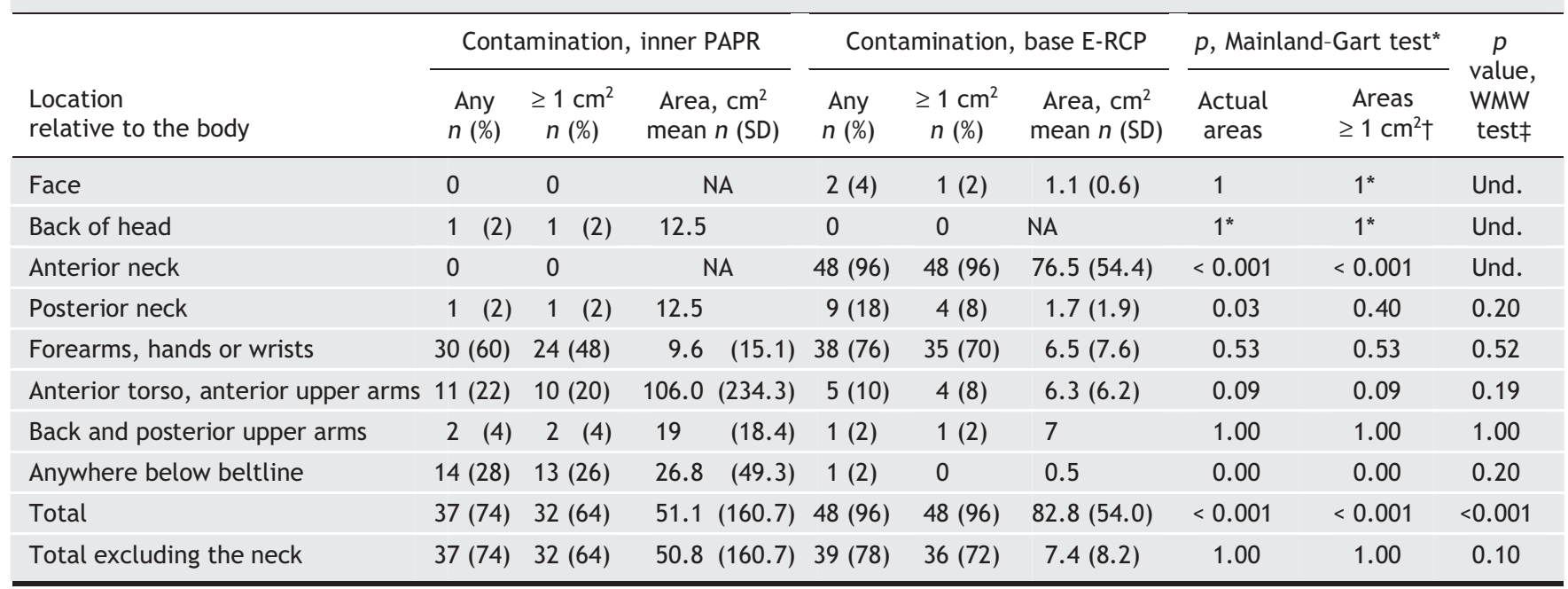

Note: $\mathrm{E}-\mathrm{RCP}=$ enhanced respiratory and contact precautions, $\mathrm{PAPR}=$ powered air-purifying respirator, WMW $=$ Wilcoxon-Mann-Whitney test, NA $=$ not applicable, Und. = undefined.

*Except where McNemar's test was used, as indicated with asterisks. The Mainland-Gart test was used when contamination occurred during both periods. McNemar's test was substituted when only 1 period had any contamination. Neither test was applied when there was no contamination in either period.

†Repeat analysis, in which areas $<1 \mathrm{~cm}^{2}$ in area were counted as $0 \mathrm{~cm}^{2}$.

¥The Wilcoxon-Mann-Whitney test was used to compare the area of contamination for subjects with some contamination. Since these contaminated subjects were different for each system, a test for paired data was not used. 
Table 5: Donning and removal protocol violations

Task error

No.

\section{Powered air-purifying respirator system}

Donning errors

Failure to remove jewelry, pager, nametag or stethoscope

Failure to tuck long shoelaces into shoes

Error in application of goggles

Error in application of boot covers

Failure to zip up coveralls or put hood over head

Error in application of second boot covers

Error in application of HEPA filter unit

Failure to tuck extra belt into waistband

Torn suit

Removal errors

Error in removal of second pair of gloves

Error in removal of first pair of boot covers

Error in removal of third pair of gloves

Failure to disinfect hands, wrists or lower arms

Torn suit

\section{Enhanced respiratory and contact precautions}

Donning errors

Error in application of bouffant hair-cover

Error in application of surgical gown

Removal errors

Error in removal of first pair of gloves

Failure to don or error in application of a third pair of gloves

Error in removal of face shield

Error in removal of hair-cover

Error in removal of third pair of gloves

Note: $\mathrm{E}-\mathrm{RCP}=$ enhanced respiratory and contact precautions, $\mathrm{PAPR}=$ powered air-purifying respirator, HEPA = high-efficiency particulate air.

It was not the intent of our study to examine respiratory contamination that might defeat the $\mathrm{N}_{95}$ (or equivalent) mask that forms a part of both of these personal protective systems.

Despite these limitations, this study provides us with a valuable first step in the examination of the relative effectiveness of protective clothing systems used by HCWs. Future attempts to optimize HCW-protective clothing should involve efforts to find a solution to the different levels of protection associated with specific systems and to improve decontamination procedures. The strengths and limitations of each protective system need to be considered when recommendations are made about which choice of system, donning and removal procedures, and decontamination procedures are optimal for specific clinical situations.

\section{This article has been peer reviewed.}

From the Department of Anesthesiology (Zamora, Murdoch, Simchison), Queen's University, and the Clinical Research Centre (Day), Kingston General Hospital, Kingston, Ont.
Competing interests: None declared.

Contributors: All of the authors contributed substantially to the conception and design of the study, and the acquisition, analysis and interpretation of the data. All of the authors drafted the article, reviewed it for important intellectual content and gave final approval of the version to be published.

Acknowledgements: We thank Cynthia Philips, Derry Thibeault and Nathan Luyt for their contributions to the study.

This study was funded by the Physicians' Services Incorporated Foundation and the Clinical Teachers' Association of Queen's University.

\section{REFERENCES}

I. Weber SG, Bottei E, Cook R, et al. SARS, emerging infections, and bioterrorism preparedness. Lancet Infect Dis 2004;4:483-4.

2. Ontario Ministry of Health and Long-Term Care. Directive to all Ontario health care facilities/settings for high risk aerosol generating procedures under outbreak conditions. Directive HRo4-I3 April I5, 2004. Available: www.health.gov.on.ca/english /providers/program/pubhealth/sars/docs/docs3/dir_aerosol_outbreak_04I504.pdf (accessed 2006 May 17).

3. Centers for Disease Control and Prevention. Supplement I: Infection control in healthcare, home, and community settings. Available: www.cdc.gov/ncidod/sars /guidance/I/index.htm (accessed 2006 May 17 ).

4. Health Canada. Cluster of severe acute respiratory syndrome cases among protected health care workers - Toronto, April 2003. Can Commun Dis Rep 2003;29:93-7.

5. Christian MD, Loutfy M, McDonald LC, et al. Possible SARS Coronavirus transmission during cardiopulmonary resuscitation. Emerg Infect Dis 2004;10:287-93.

6. Poutanen SM, McGeer AJ. Transmission and control of SARS. Curr Infect Dis Rep 2004;6:220-7.

7. Puro V, Nicastri E. SARS and the removal of personal protective equipment [letter]. CMAJ 2004;170(6):930.

8. Wong TK, Chung JW, Li Y, et al. Effective personal protective clothing for health care workers attending patients with severe acute respiratory syndrome. Am J Infect Control 2004;32:90-6.

9. Cooper DM, Charles D, Durnell AJ, et al. Assessment of personal protective equipment used for facial mucocutaneous exposure protection in nonhuman primate areas. Lab Anim (NY) 2005;34:49-53.

Io. Fleiss JL. The design and analysis of clinical experiments. New York: Wiley; 1986.

II. Gart JJ. An exact test for comparing matched proportions in crossover designs. Biometrika I969; 56(I):75-80.

I2. Nam J. 2 tests for comparing matched proportions. Biometrics I971;27:945-59.

Correspondence to: Dr. Jorge Zamora, Department of Anesthesiology, Queen's University, Kingston General Hospital, 76 Stuart St., Kingston ON K7L 2V7; fax: 613 548-1375; zamoraj@kgh.kari.net

\section{Editor's take}

- We have known for decades that microbes, given enough time, can pass through protective gowns. With the emergence of novel life-threatening pathogens, prevention of this transmission has become urgent. How much additional protection is required will depend on the size of the particular microbe and whether it is transmitted by contact, droplet or aerosol.

- In this simulation study examining degrees of protection against aerosol contamination, the investigators found that ordinary gown, gloves and mask were inadequate barriers. Furthermore, breaches in technique can result in selfcontamination even with a highly protective system.

Implications for practice: During an aerosol-transmitted outbreak, use of the right kind of protective outfit and the correct technique for its use and removal will be critical to prevent disease transmission. 